\title{
SUPERCUSPIDAL RAMIFICATION OF MODULAR ENDOMORPHISM ALGEBRAS
}

\author{
SHALINI BHATTACHARYA AND EKNATH GHATE
}

(Communicated by Matthew A. Papanikolas)

\begin{abstract}
The endomorphism algebra $X_{f}$ attached to a non-CM primitive cusp form $f$ of weight at least two is a 2-torsion element in the Brauer group of a number field $F$. We give formulas for the ramification of $X_{f}$ locally at primes lying above the odd supercuspidal primes of $f$. We show that the local Brauer class is determined by the underlying local Galois representation together with an auxiliary Fourier coefficient.
\end{abstract}

\section{INTRODUCTION}

Let $f=\sum_{n=1}^{\infty} a_{n} q^{n} \in S_{k}(N, \epsilon)$ be a primitive non-CM cusp form of weight $k \geq 2$ and level $N \geq 1$. Let $M_{f}$ denote the abelian variety $(k=2)$ or the Grothendieck motive $(k>2)$ attached to $f$. The $\mathbb{Q}$-algebra of endomorphisms of $M_{f}$ is denoted by

$$
X=X_{f}:=\operatorname{End}_{\overline{\mathbb{Q}}}\left(M_{f}\right) \otimes_{\mathbb{Z}} \mathbb{Q} .
$$

The Hecke field $E=\mathbb{Q}\left(a_{n}\right)$ is either a totally real or CM number field. Let $F$ denote the subfield of $E$ generated by all the elements of the form $a_{n}^{2} \epsilon^{-1}(n)$ with $(n, N)=1$. Then $F$ is a totally real number field and may be thought of as the Hecke field of the adjoint lift of $f$. The algebra $X$ has the structure of a crossed product algebra over $F$ defined in terms of the inner twists of $f$, as proved in [Ri80] and Ri81 for $k=2$, and generalized to higher weight forms in BG03] and GGQ05. The class of $X$ is a 2-torsion element in the Brauer group $\operatorname{Br}(F)$ of $F$. Ribet has asked whether one can determine the class $[X] \in \operatorname{Br}(F)$ explicitly.

The Brauer class of $X$ can be studied locally under the map $\operatorname{Br}(F) \hookrightarrow \bigoplus_{v} \operatorname{Br}\left(F_{v}\right)$, where $v$ varies over all the primes in $F$. The algebra $X_{v}:=X \otimes_{F} F_{v}$ is central simple over $F_{v}$ and its class $\left[X_{v}\right] \in{ }_{2} \operatorname{Br}\left(F_{v}\right) \cong \mathbb{Z} / 2$. When this class is trivial, $X_{v}$ is a matrix algebra over $F_{v}$ and $X_{v}$ is said to be unramified, and when it is non-trivial, $X_{v}$ is Brauer-equivalent to a quaternion division algebra over $F_{v}$ and $X_{v}$ is said to be ramified. The Brauer class of $X$ is determined by the Brauer classes of all the $X_{v}$, only finitely many of which can be non-trivial.

For $v$ lying above a prime $p$ of good reduction, a Steinberg prime $p$, or a ramified principal series prime $p$, with $a_{p} \neq 0$, the class $\left[X_{v}\right]$ is essentially determined by the parity of the slope at $p$ of the twisted adjoint lift of $f$. For instance, if $p \nmid N$, then $\left[X_{v}\right]$ is essentially determined by the $v$-adic valuation of $a_{p}^{2} \epsilon^{-1}(p) \in F^{*}$. See [BG13]. In particular, if $\rho_{f}$ is the $\ell$-adic Galois representation attached to $f$, then the $\overline{\mathbb{Q}}_{\ell}$-isomorphism class of the local representation $\left.\rho_{f}\right|_{G_{p}}$ at $p$ essentially determines

Received by the editors February 14, 2014 and, in revised form, August 11, 2014.

2010 Mathematics Subject Classification. Primary 11F30; Secondary 11F11, 11F80. 
the Brauer class $\left[X_{v}\right]$, for $\ell \neq p$. Thus, if the Fourier coefficient $a_{p} \neq 0$, then it essentially determines $\left[X_{v}\right]$.

Assume, therefore, that $a_{p}=0$. For $v$ lying above a prime $p$ of good reduction with $a_{p}=0$, the slope of the twisted adjoint lift of $f$ at $p$ is not finite. Moreover, the local $\ell$-adic Galois representation $\left.\rho_{f}\right|_{G_{p}}$ does not determine the class $\left[X_{v}\right]$, even if $\ell=p$. However, in $\left[\mathrm{BG} 13\right.$. Thm. 11] it is shown that $\left[X_{v}\right]$ is essentially determined by a Fourier coefficient $a_{p^{\dagger}} \neq 0$ at an auxiliary prime $p^{\dagger} \nmid N$. More precisely, $\left[X_{v}\right]$ is essentially determined by the $v$-adic valuation of $a_{p^{\dagger}}^{2} \epsilon^{-1}\left(p^{\dagger}\right)$, the Fourier coefficient of the twisted adjoint lift of $f$ at $p^{\dagger}$.

Assume now that $v$ lies over a prime $p$ of bad reduction with $a_{p}=0$. Since the Brauer class of $\left[X_{f}\right]$ is invariant under twisting $f$ by Dirichlet characters, we may as well assume that $f$ is $p$-minimal, in which case $p$ is a supercuspidal prime. Almost nothing is known about the ramification of $X_{v}$ in this case. Again the $\overline{\mathbb{Q}}_{\ell}$-isomorphism class of $\left.\rho_{f}\right|_{G_{p}}$ does not determine $\left[X_{v}\right]$; see example (1) in Section 8. In this paper, we obtain formulas for $\left[X_{v}\right]$ in terms of a Fourier coefficient at an auxiliary prime related to $p$, as in the good reduction case. It turns out that the $\overline{\mathbb{Q}}_{\ell}$-isomorphism class of $\left.\rho_{f}\right|_{G_{p}}$, for $\ell \neq p$, together with this coefficient, determines $\left[X_{v}\right]$ completely. This solves the problem of determining the Brauer classes at the odd supercuspidal primes (under an extra hypothesis in the level 0 case).

\section{Statement of Results}

Throughout this article, $p$ will denote an odd prime. Let $f \in S_{k}(N, \epsilon)$ be a non-CM primitive cusp form of weight $k \geq 2$, level $N$ and nebentypus $\epsilon$. We write $N=p^{N_{p}} N^{\prime}$, such that $p \nmid N^{\prime}$, and $\epsilon=\epsilon_{p} \cdot \epsilon^{\prime}$, where $\epsilon_{p}$ is a Dirichlet character of conductor $p^{C_{p}}$ for some $C_{p} \leq N_{p}$, and the conductor of $\epsilon^{\prime}$ divides $N^{\prime}$. If $f$ is $p$-minimal and if $C_{p}<N_{p} \geq 2$, then $p$ is a supercuspidal prime for $f$ and $a_{p}=0$. Let $(K, \theta)$ be an admissible pair attached to $f$ at $p$, where $K$ is a quadratic extension of $\mathbb{Q}_{p}$ and $\theta$ is a continuous character of $K^{*}$. The prime $p$ is called a ramified (or unramified) supercuspidal prime for $f$, if $K \mid \mathbb{Q}_{p}$ is a ramified (or unramified) extension. When $C_{p}<N_{p}=2$, then $p$ is an unramified supercuspidal prime and $\theta$ is a tamely ramified character of $K^{*}$. The corresponding local automorphic representation has level 0 , and we say $p$ is supercuspidal of level 0 . When $K \mid \mathbb{Q}_{p}$ is unramified, we let $s$ denote a fixed primitive $\left(p^{2}-1\right)$-th root of unity in $K^{*}$. For any quadratic field extension $K_{1} \mid K_{2}$, and for an arbitrary $x \in K_{2}^{*}$, let

$$
\left(x, K_{1} \mid K_{2}\right):= \begin{cases}-1, & \text { if } x \notin N_{K_{1} \mid K_{2}}\left(K_{1}\right)^{*}, \\ 1, & \text { otherwise. }\end{cases}
$$

For a prime $v$ in $F$ lying above $p$, let $f_{v}:=f\left(F_{v} \mid \mathbb{Q}_{p}\right)$ be the residue degree and let $e_{v}:=e\left(F_{v} \mid \mathbb{Q}_{p}\right)$ be the ramification index. Let $v: F_{v}^{*} \rightarrow \mathbb{Z}$ be the standard surjective valuation.

Since $f$ is non-CM, there exist infinitely many primes with non-zero Fourier coefficient in any congruence class modulo $N$. Let us choose two primes $p^{\prime}$ and $p^{\prime \prime}$, both coprime to $N$, satisfying:

(*) $p^{\prime} \equiv 1 \bmod p^{N_{p}}, p^{\prime} \equiv p \bmod N^{\prime}$ and $a_{p^{\prime}} \neq 0$.

$(* *) p^{\prime \prime} \equiv 1 \bmod N^{\prime}, p^{\prime \prime}$ has order $(p-1)$ in $\left(\mathbb{Z} / p^{N_{p}} \mathbb{Z}\right)^{*}$ and $a_{p^{\prime \prime}} \neq 0$.

The Fourier coefficients of the twisted adjoint lift of $f$ at $p^{\prime}$ and $p^{\prime \prime}$, namely $a_{p^{\prime}}^{2} \epsilon^{-1}\left(p^{\prime}\right)$ and $a_{p^{\prime \prime}}^{2} \epsilon^{-1}\left(p^{\prime \prime}\right)$, give elements of $F^{*} /\left(F^{*}\right)^{2}$ which do not depend on 
the choice of $p^{\prime}$ and $p^{\prime \prime}$. Let us write

$$
\left[X_{v}\right] \sim \begin{cases}-1, & \text { if } X_{v} \text { is ramified } \\ 1, & \text { if } X_{v} \text { is unramified }\end{cases}
$$

With the notation as above, our main result is as follows.

Theorem 2.1. Let $v$ be a prime of $F$ lying over a prime $p \neq 2$.

(a) If $p$ is an unramified supercuspidal prime, and $\theta(s)+\theta(s)^{p} \neq 0$ if the p-minimal twist of $f$ is of level 0 , then

$$
\left[X_{v}\right] \sim(-1) f_{v} \cdot v\left(a_{p^{\prime}}^{2} \epsilon^{-1}\left(p^{\prime}\right)\right) .
$$

(b) If $p \equiv 1 \bmod 4$ is a ramified supercuspidal prime, then

$$
\left[X_{v}\right] \sim(-1)^{f_{v} \cdot v\left(a_{p^{\prime}}^{2} \epsilon^{-1}\left(p^{\prime}\right)\right) .}
$$

(c) If $p \equiv 3 \bmod 4$ is a ramified supercuspidal prime, then

$$
\left[X_{v}\right] \sim\left((-1)^{k} \cdot a_{p^{\prime \prime}}^{2} \epsilon^{-1}\left(p^{\prime \prime}\right), K F_{v} \mid F_{v}\right) .
$$

Remark. One reason behind the similarity and simplicity of the formulas in (a) and (b) above is that in both cases the extension $K F_{v} \mid F_{v}$ turns out to be unramified, and hence the symbol $\left(a_{p^{\prime}}^{2} \epsilon^{-1}\left(p^{\prime}\right), K F_{v} \mid F_{v}\right)=(-1)^{v\left(a_{p^{\prime}}^{2} \epsilon^{-1}\left(p^{\prime}\right)\right)}$. On the other hand, we will see that in case (c) the extension $K F_{v} \mid F_{v}$ can be ramified.

We list some interesting consequences of the theorem.

Corollary 2.2. Suppose we are in case (a) or (b) of the theorem. If either

(1) $N^{\prime}=1$ (i.e., $N$ is a prime power) or if $p$ has odd order in $\left(\frac{\mathbb{Z}}{N^{\prime} \mathbb{Z}}\right)^{*}$ or

(2) $v \nmid \operatorname{disc}(E \mid F)$,

then $X_{v}$ is unramified.

Corollary 2.3. Suppose we are in case (a) or (c) of the theorem. If $K \subseteq F_{v}$, then $X_{v}$ is unramified.

We will show that $(p-1)$ divides $2 e_{v}$ if $p$ is a ramified supercuspidal prime.

Corollary 2.4. Suppose we are in case (b) of the theorem. If $K=\mathbb{Q}_{p}(\sqrt{p})$, then

$$
\left[X_{v}\right] \sim \epsilon_{p}(-1)^{2\left[F_{v}: \mathbb{Q}_{p}\right] /(p-1)} .
$$

\section{Endomorphism ALGEBRA AND ITS COCYCLE CLASS}

Let $f$ and $E$ be as above. Then $\operatorname{Aut}(E)$ contains an abelian subgroup defined by $\Gamma:=\left\{\gamma \in \operatorname{Aut}(E) \mid \exists\right.$ a Dirichlet character $\chi_{\gamma}$ such that $\left.a_{p}^{\gamma}=a_{p} \cdot \chi_{\gamma}(p), \forall p \nmid N\right\}$. The subfield of $E$ fixed by $\Gamma$ equals the field $F$ mentioned in Section 1 . As $f$ is non-CM, for each $\gamma \in \Gamma$, the character $\chi_{\gamma}$ is unique and is called an 'inner twist' of $f$. For a fixed $g \in G_{\mathbb{Q}}$, the map $\gamma \mapsto \chi_{\gamma}(g)$ is a 1-cocycle. By Hilbert's theorem $90, \exists \alpha(g) \in E^{*}$ such that for all $\gamma \in \Gamma$,

$$
\chi_{\gamma}(g)=\alpha(g)^{\gamma-1} \text {. }
$$

Thus we get a well-defined continuous character $\tilde{\alpha}: G_{\mathbb{Q}} \rightarrow E^{*} / F^{*}$, sending each $g$ to $\alpha(g) \bmod F^{*}$. Let $\rho_{f}$ denote the $\lambda$-adic representation attached to $f$ by Deligne, for some prime $\lambda \mid \ell$ of $E$. The following result of Ribet [Ri04, Thm. 5.5] for $k=2$, holds for all weights $k \geq 2$. 
Proposition 3.1. Let $\alpha$ be any lift of the character $\tilde{\alpha}$ to $E^{*}$. Then

(1) For all $g \in G_{\mathbb{Q}}, \alpha^{2}(g) \equiv \epsilon(g) \bmod F^{*}$.

(2) For all $g \in G_{\mathbb{Q}}, \alpha(g) \equiv \operatorname{Trace}\left(\rho_{f}(g)\right) \bmod F^{*}$, provided that the trace is non-zero.

For any continuous lift $\alpha$ of $\tilde{\alpha}$ to $E^{*}$, the 2-cocycle $c_{\alpha}$ defined by $c_{\alpha}(g, h)=$ $\alpha(g) \alpha(h) \alpha^{-1}(g h)$ gives a 2 -torsion class $\left[c_{\alpha}\right]$ in $\mathrm{H}^{2}\left(G_{F}, \bar{F}^{*}\right) \cong \operatorname{Br}(F)$. The class $\left[c_{\alpha}\right] \in \mathrm{H}^{2}\left(G_{F}, \bar{F}^{*}\right)$ corresponds to the global Brauer class $\left[X_{f}\right] \in \operatorname{Br}(F)$. Similarly the local Brauer class $\left[X_{v}\right]$ is determined by the restriction $\left[\left.c_{\alpha}\right|_{G_{F_{v}}}\right] \in \mathrm{H}^{2}\left(G_{F_{v}}, \bar{F}_{v}^{*}\right)$, for any prime $v$ of $F$.

Let $G_{p}$ by the decomposition group at $p$. If $p$ is a supercuspidal prime, then the local Galois representation $\left.\rho_{f}\right|_{G_{p}}$ is induced from a character of $G_{K}$ for some quadratic extension $K$ of $\mathbb{Q}_{p}$. This implies that for any lift $g \in G_{p}$ of the generator of $\operatorname{Gal}\left(K \mid \mathbb{Q}_{p}\right)$, the trace in part (2) always vanishes, so one cannot use Proposition 3.1 to compute $\alpha(g)$. In fact, the auxiliary primes $p^{\prime}$ and $p^{\prime \prime}$ introduced in the previous section are chosen in such a way that there is a lift $g$ for which $\alpha(g) \equiv a_{p^{\prime}}$ or $a_{p^{\prime \prime}} \bmod F^{*}$.

\section{Galois Representations and Langlands correspondence}

The pair $(K, \theta)$ is called an 'admissible pair' if $K$ is a quadratic extension of $\mathbb{Q}_{p}$ and $\theta: K^{*} \rightarrow \overline{\mathbb{Q}}^{*} \subseteq \overline{\mathbb{Q}}_{\ell}^{*}$ is a (continuous) character of $K^{*}$ satisfying

(1) $\theta$ does not factor through the norm map $N_{K \mid \mathbb{Q}_{p}}: K^{*} \rightarrow \mathbb{Q}_{p}^{*}$,

(2) if $K \mid \mathbb{Q}_{p}$ is ramified, then even $\left.\theta\right|_{U_{K}^{(1)}}$ does not factor through $N_{K \mid \mathbb{Q}_{p}}$.

Here $U_{K}^{(i)}$ denotes the $i$-th step in the standard filtration of the units of $K$. Two pairs $\left(K_{1}, \theta_{1}\right)$ and $\left(K_{2}, \theta_{2}\right)$ are equivalent if there is a $\mathbb{Q}_{p}$-isomorphism $\iota: K_{1} \rightarrow K_{2}$ with $\theta_{2} \circ \iota=\theta_{1}$. The equivalence classes of admissible pairs are in bijection with $\overline{\mathbb{Q}}_{\ell}$-isomorphism classes of irreducible 2-dimensional representations of $G_{p}$ as well as with $\overline{\mathbb{Q}}_{\ell}$-isomorphism classes of supercuspidal representations of $\mathrm{GL}_{2}\left(\mathbb{Q}_{p}\right)$ (for $p \neq 2$ ).

If $p$ is a supercuspidal prime for $f$, then $\left.\rho_{f}\right|_{G_{p}}$ is absolutely irreducible. We call $(K, \theta)$ an admissible pair attached to $f$ at $p$ if

$$
\left.\rho_{f}\right|_{G_{p}} \sim \operatorname{Ind}_{G_{K}}^{G_{p}} \theta .
$$

Given a supercuspidal prime $p(\neq 2)$ for $f$, the quadratic extension $K$ is unique, though it is not easy to determine the admissible pair $(K, \theta)$ attached to $f$ at $p$ explicitly; see [LW12].

For $L \mid \mathbb{Q}_{p}$ a finite extension and an $\ell$-adic representation $\rho$ of $G_{L}$, let $c(\rho)$ be the conductor of the corresponding representation of the Weil group of $L$. It equals the Artin conductor of a suitable unramified twist of $\rho$. For the character $\theta$ of $G_{K}$, it equals the usual $c(\theta)=\min \left\{i:\left.\theta\right|_{U_{K}^{(i)}} \equiv 1\right\}$. By CF10, Prop. 4(b), §4.3, Ch. $6]$, we get $c\left(\operatorname{Ind}_{G_{K}}^{G_{p}} \theta\right)=v_{p}\left(\delta\left(K \mid \mathbb{Q}_{p}\right)\right)+f\left(K \mid \mathbb{Q}_{p}\right) c(\theta)$, where $v_{p}$ is the normalized valuation on $\mathbb{Q}_{p}^{*}, \delta\left(K \mid \mathbb{Q}_{p}\right)$ stands for the discriminant, and $f\left(K \mid \mathbb{Q}_{p}\right)$ is the residue degree. Applying this formula in our setting, we get

$$
N_{p}= \begin{cases}2 c(\theta), & \text { if } K=\mathbb{Q}_{p^{2}} \text { is unramified } \\ 1+c(\theta), & \text { if } K \mid \mathbb{Q}_{p} \text { is ramified }\end{cases}
$$


Thus if $K=\mathbb{Q}_{p^{2}}$, then $N_{p}$ is even. A newform $f$ of level $N=p^{N_{p}} N^{\prime}$ is said to be $p$-minimal if $N_{p}$ is the smallest among all possible twists $f \otimes \chi$ of $f$ by Dirichlet characters $\chi$. If $f$ is a $p$-minimal form for which $p$ is a supercuspidal prime, then by [AL78, Thms. 4.3, 4.3'], $C_{p} \leq\left\lfloor N_{p} / 2\right\rfloor \geq 1$; moreover, if $N_{p}$ is even, then $K \mid \mathbb{Q}_{p}$ is unramified.

While proving the $\ell$-adic local Langlands correspondence for $\mathrm{GL}_{2}\left(\mathbb{Q}_{p}\right)$, the 'supercuspidals of level zero' are always treated separately. They are exactly the supercuspidal representations attached to some admissible pairs $(K, \theta)$ with $c(\theta)=$ 1 , and hence $N_{p}=2$. By the definition of an admissible pair, $c(\theta)=1$ forces $K$ to be unramified. For the exact definition of the level of a supercuspidal representation we refer to $[\mathrm{BH} 06, \S 12.6]$. The next lemma will be used to investigate the case of supercuspidal primes of positive level.

Let $\sigma$ be any lift of the generator of $\operatorname{Gal}\left(K \mid \mathbb{Q}_{p}\right)$ to $G_{p}$, and let $\theta^{\sigma}$ denote the conjugate character of $\theta$ by $\sigma$. Then we have

Lemma 4.1. Let $p$ be a supercuspidal prime for $f$ with $N_{p} \geq 3$ and suppose $\epsilon$ is tamely ramified at $p$. Then there is an element $\tau \in I_{w}(K)$, the wild inertia group of $K$, satisfying:

(1) $\theta(\tau)=\zeta_{p}$ and $\theta^{\sigma}(\tau)=\zeta_{p}^{-1}$, where $\zeta_{p}$ is some primitive $p$-th root of unity.

(2) $\alpha(\tau) \equiv\left(\zeta_{p}+\zeta_{p}^{-1}\right) \equiv 1 \bmod F^{*}$.

Thus $\mathbb{Q}\left(\zeta_{p}+\zeta_{p}^{-1}\right) \subseteq F$. In particular, $(p-1) / 2$ divides $e\left(F_{v} \mid \mathbb{Q}_{p}\right)=e_{v}$, for all $v \mid p$. Proof. By equation (2) above, $N_{p} \geq 3 \Rightarrow c(\theta)>1$, so $\left.\theta\right|_{U_{K}^{(1)}} \neq 1$. So $\exists \tau \in I_{w}(K)$ with $\theta(\tau)=\zeta_{p}$, for some primitive $p$-th root of unity $\zeta_{p}$. As $\epsilon_{p}$ is tame, $\epsilon(\tau)=1$, and $\chi_{\gamma}^{2}(\tau)=\epsilon(\tau)^{\gamma-1}=1$, for all $\gamma \in \Gamma$. But since $\tau$ is an element of a pro- $p$ group, and $p$ is odd, we conclude that $\chi_{\gamma}(\tau)=1$, for all $\gamma \in \Gamma$. In other words, $\alpha(\tau) \equiv 1$ $\bmod F^{*}$.

Both $\theta(\tau)$ and $\theta^{\sigma}(\tau)$ have some $p$-power order. The sum of two roots of unity of odd order cannot be zero. Hence by Proposition 3.1, $\alpha(\tau) \equiv\left(\theta+\theta^{\sigma}\right)(\tau) \equiv 1$ $\bmod F^{*}$. Since $F$ is totally real, $\theta(\tau)$ and $\theta^{\sigma}(\tau)$ are two roots of unity whose sum is a non-zero real number. Hence they are complex conjugates.

Lemma 4.2. If $p \neq 2$ is a supercuspidal prime of level $>0$ for a p-minimal $f$, then $F$ contains $\mathbb{Q}\left(\zeta_{p}+\zeta_{p}^{-1}\right)$ and $e_{v}$ is a multiple of $(p-1) / 2$, for each prime $v$ in $F$ above $p$.

Proof. Since $p$ is odd, we can twist $f$ by a suitable character to make the nebentypus tame at $p$, without changing the field $F$. As $f$ was $p$-minimal, $N_{p} \geq 3$ must hold, even after twisting. Now apply the previous lemma.

We know that $\operatorname{det}\left(\rho_{f}\right)=\chi_{\ell}^{k-1} \epsilon$, where $\chi_{\ell}$ is the $\ell$-adic cyclotomic character. The nebentypus character $\epsilon$ may be adelized as follows. For $x \in \mathbb{Q}_{p}^{*}$, let $[x]$ denote the corresponding element $(1, \cdots, x, \cdots, 1) \in \mathbb{A}_{\mathbb{Q}}^{*}$. Then the idèlic character $\epsilon$, restricted to $\mathbb{Q}_{p}^{*}$, is given by the following formula. For any $m \in \mathbb{Z}$ and $u \in \mathbb{Z}_{p}^{*}$,

$$
\epsilon\left(\left[p^{m} u\right]\right)=\epsilon^{\prime}(p)^{m} \epsilon_{p}(u)^{-1} \text {. }
$$

The Galois character $\left.\epsilon\right|_{G_{p}}$ is also determined by this formula via the norm residue map of class field theory, which maps $\mathbb{Q}_{p}^{*} \subseteq \mathbb{A}_{\mathbb{Q}}^{*}$ onto a dense subset of the decomposition group $G_{p}$ at $p$.

The local Langlands correspondence for $\mathrm{GL}_{2}$ is described using the theory of admissible pairs. In BH06, for any admissible pair $(K, \theta)$ the authors construct an 
automorphic representation $\pi_{\theta}$ with central character $\theta$, as well as a 2-dimensional Galois representation, say $\rho_{\theta}$, both unique up to isomorphism. Let $p$ be a supercuspidal prime for $f$ and $(K, \theta)$ be an admissible pair attached to $f$ at $p$. There exists a character $\Delta_{\theta}$ of $K^{*}$ such that $\left(K, \theta \Delta_{\theta}\right)$ is also an admissible pair, and the supercuspidal representation $\pi_{\theta \Delta_{\theta}}$ of $\mathrm{GL}_{2}\left(\mathbb{Q}_{p}\right)$ is in Langlands correspondence with $\left.\rho_{f}\right|_{G_{p}}$. Equating the central character with the determinant on the Galois side, we get

$$
\left.\left(\theta \Delta_{\theta}\right)\right|_{\mathbb{Q}_{p}^{*}}=\left.\left(\chi_{\ell}^{k-1} \epsilon\right)\right|_{\mathbb{Q}_{p}^{*}}
$$

We refer to $\mathrm{BH} 06$, §34] for the explicit description of $\Delta_{\theta}$. But let us mention here that it is a quadratic character unless we are in case (c) of Theorem 2.1 (in which case $\Delta_{\theta}$ has order 4 ) and that $\left.\Delta_{\theta}\right|_{\mathbb{Q}_{p}^{*}}$ is always the unique non-trivial character factoring through $N_{K \mid \mathbb{Q}_{p}}\left(K^{*}\right)$. Using the equations above, we get that for any $u \in \mathbb{Z}_{p}^{*}$

$$
\theta(u)= \begin{cases}\epsilon_{p}(u)^{-1}, & \text { if } K=\mathbb{Q}_{p^{2}}, \\ \left(u, K \mid \mathbb{Q}_{p}\right) \cdot \epsilon_{p}(u)^{-1}, & \text { if } K \mid \mathbb{Q}_{p} \text { is ramified }\end{cases}
$$

\section{LOCAL SYMBOLS}

We use the same notation as in the previous section. Consider the nebentypus $\epsilon$ as a Galois character, and fix a square root $\sqrt{\epsilon(g)}$, for each $g \in G_{\mathbb{Q}}$. For all $\gamma \in G_{F}$, there exists a unique quadratic (of order 1 or 2) Dirichlet character $\psi_{\gamma}$, such that $\forall g \in G_{\mathbb{Q}}$,

$$
\chi_{\gamma}(g)=\sqrt{\epsilon(g)}^{\gamma-1} \psi_{\gamma}(g) .
$$

Note that here $\chi_{\gamma}$ means the inner twist corresponding to the image of $\gamma \in G_{F}$ in its quotient $\Gamma=\operatorname{Gal}(E \mid F)$. For any $\gamma \in G_{F}$, let $t_{\gamma}$ denote the fundamental discriminant corresponding to the character $\psi_{\gamma}$. The set $A=\left\{\psi_{\gamma}: \gamma \in G_{F}\right\}$ is an elementary 2-group. Let $\Gamma_{0} \subseteq G_{F}$ be a fixed subset such that $\left\{\psi_{\gamma}: \gamma \in \Gamma_{0}\right\}$ forms a basis for the group $A$. For each $\gamma \in \Gamma_{0}$, choose a square-free positive integer $n_{\gamma}$ prime to $N$, with $a_{n_{\gamma}} \neq 0$, and such that for all $\gamma^{\prime} \in \Gamma_{0}$,

$$
\psi_{\gamma^{\prime}}\left(n_{\gamma}\right)= \begin{cases}-1, & \text { if } \gamma^{\prime}=\gamma \\ 1, & \text { otherwise }\end{cases}
$$

For each $n_{\gamma}$, set $z_{n_{\gamma}}:=a_{n_{\gamma}}^{2} \epsilon^{-1}\left(n_{\gamma}\right) \in F^{*}$. Let $v$ be a prime in $F$ above some odd prime $p$. Let $\left[c_{\epsilon}\right]_{v}$ denote the class of the cocycle $c_{\epsilon} \in \mathrm{Z}^{2}\left(G_{F_{v}},\{ \pm 1\}\right)$ defined by $c_{\epsilon}(g, h)=\sqrt{\epsilon(g)} \sqrt{\epsilon(h)} \sqrt{\epsilon(g h)}^{-1}$. It follows from Qu98 that

$$
\left[c_{\epsilon}\right]_{v} \sim \epsilon_{v}(-1)=\epsilon_{p}(-1)^{\left[F_{v}: \mathbb{Q}_{p}\right]} .
$$

For any two elements $a, b \in F_{v}^{*}$, let us write $a=\pi_{v}^{v(a)} \cdot a^{\prime}$ and $b=\pi_{v}^{v(b)} \cdot b^{\prime}$, where $\pi_{v}$ is a uniformizer in $F_{v}$. Then the local symbol $(a, b)_{v}$, which is independent of the choice of $\pi_{v}$, is given by the following equation:

$$
(a, b)_{v}=(-1)^{v(a) v(b)(N v-1) / 2} \cdot\left(\frac{b^{\prime}}{v}\right)^{v(a)} \cdot\left(\frac{a^{\prime}}{v}\right)^{v(b)},
$$

where $(\dot{v})$ is the standard quadratic residue symbol in the residue field of $F_{v}$. The next formula expressing the local Brauer class in terms of symbols follows from GGQ05, Thm. 4.1]. 
Theorem 5.1. The local Brauer class is given by

$$
\left[X_{v}\right] \sim\left[c_{\epsilon}\right]_{v} \otimes \bigotimes_{\gamma \in \Gamma_{0}}\left(z_{n_{\gamma}}, t_{\gamma}\right)_{v}
$$

Note that each $t_{\gamma}$ must divide $N$. If $p$ divides $N, p^{*}:=\left(\frac{-1}{p}\right) \cdot p$ may or may not divide $t_{\gamma}$ for a given $\gamma \in \Gamma_{0}$. For a fixed set $\Gamma_{0}$ as above, let $S:=\left\{t_{\gamma}: \gamma \in \Gamma_{0}\right\}$. Let us write $S$ as a disjoint union of three sets: $S=S_{p} \cup S^{-} \cup S^{+}$, where

$$
\begin{gathered}
S_{p}=\left\{t_{\gamma} \in S: p^{*} \mid t_{\gamma}\right\}, \quad S^{-}=\left\{t_{\gamma} \in S \backslash S_{p}:\left(\frac{t_{\gamma}}{p}\right)=-1\right\}, \\
S^{+}=\left\{t_{\gamma} \in S \backslash S_{p}:\left(\frac{t_{\gamma}}{p}\right)=1\right\} .
\end{gathered}
$$

The statement of Theorem 5.1 is true for any choice of $\Gamma_{0} \subseteq G_{F}$, as long as $\left\{\psi_{\gamma}: \gamma \in \Gamma_{0}\right\}$ forms a basis of the elementary 2-group $A$. If $S_{p}$ consists of more than one element, we can choose some $t_{\gamma_{0}} \in S_{p}$ and multiply the (quadratic) characters corresponding to all other elements of $S_{p}$ by $\psi_{\gamma_{0}}$, to construct a new basis of $A$, for which $S_{p}$ is a singleton. Similarly we can multiply $\psi_{\gamma_{0}}$ by a character corresponding to any element of $S^{-}$, if necessary, and then assume that $\Gamma_{0}$ satisfies the following two conditions:

(a) If $S_{p} \neq \emptyset$, then it is a singleton; denote the unique element of $S_{p}$ by $t_{\gamma_{0}}$.

(b) If $S_{p}=\left\{t_{\gamma_{0}}\right\} \neq \emptyset$ and $S^{-} \neq \emptyset$ too, then $\left(\frac{\tilde{t}_{\gamma_{0}}}{p}\right)=1$, where $\tilde{t}_{\gamma_{0}}:=t_{\gamma_{0}} / p^{*}$.

When $S_{p} \neq \emptyset$, we split the symbol $\left(z_{n_{\gamma_{0}}}, t_{\gamma_{0}}\right)_{v}$ involved in the statement of Theorem 5.1 into a $p$-part and a prime-to- $p$ part

$$
\left(z_{n_{\gamma_{0}}}, t_{\gamma_{0}}\right)_{v}=\left(z_{n_{\gamma_{0}}}, p^{*}\right)_{v} \cdot\left(\frac{\tilde{t}_{\gamma_{0}}}{v}\right)^{v\left(z_{n_{\gamma_{0}}}\right)} .
$$

Let $p^{\prime}$ be as in Section 2, satisfying $(*)$. Then the following general lemma computes the prime-to- $p$ part of the formula for $\left[X_{v}\right]$ in Theorem [5.1.

Lemma 5.2. If $\Gamma_{0}$ satisfies the conditions (a) and (b) above, then we have

$$
\left(\frac{\tilde{t}_{\gamma_{0}}}{v}\right)^{v\left(z_{n_{0}}\right)} \otimes \bigotimes_{t_{\gamma} \in S \backslash S_{p}}\left(z_{n_{\gamma}}, t_{\gamma}\right)_{v}=(-1)^{f_{v} \cdot v\left(a_{p^{\prime}}^{2} \epsilon^{-1}\left(p^{\prime}\right)\right)} .
$$

(If $S_{p}=\emptyset$, then the first symbol on the left hand side is assumed to be 1.)

Proof. Note that if $t_{\gamma} \notin S_{p}$, then $v\left(t_{\gamma}\right)=0$, and hence $\left(z_{n_{\gamma}}, t_{\gamma}\right)_{v}=\left(\frac{t_{\gamma}}{v}\right)^{v\left(z_{n_{\gamma}}\right)}=$ $\left(\frac{t_{\gamma}}{p}\right)^{f_{v} \cdot v\left(z_{n_{\gamma}}\right)}$, by equation (8).

First we consider the case where $S^{-}=\emptyset$. Hence $S \backslash S_{p}=S^{+}$, and so $\left(z_{n_{\gamma}}, t_{\gamma}\right)_{v}=$ $\left(\frac{t_{\gamma}}{p}\right)^{f_{v} \cdot v\left(z_{n_{\gamma}}\right)}=1$, for all $t_{\gamma} \in S \backslash S_{p}$. Thus the left hand side of (9) reduces to just the first symbol, which is $\left(\frac{\tilde{t}_{\gamma_{0}}}{v}\right)^{v\left(z_{n_{0}}\right)}=\left(\frac{\tilde{t}_{\gamma_{0}}}{p}\right)^{f_{v} \cdot v\left(z_{n_{0}}\right)}$. If either $S_{p}=\emptyset$ or if $S_{p}=\left\{t_{\gamma_{0}}\right\}$ with $\left(\frac{\tilde{t}_{\gamma_{0}}}{p}\right)=1$, then we use $(*)$ and the condition (a) on $\Gamma_{0}$ 
to check that $\psi_{\gamma}\left(p^{\prime}\right)=\left(\frac{t_{\gamma}}{p^{\prime}}\right)=1, \forall \gamma \in \Gamma_{0}$. Hence $\psi_{\gamma}\left(p^{\prime}\right) \stackrel{\text { 卢 }}{=}\left(\frac{\chi_{\gamma}\left(p^{\prime}\right)}{\sqrt{\epsilon\left(p^{\prime}\right)}}\right)^{\gamma-1}=$ $\left(\frac{a_{p^{\prime}}}{\sqrt{\epsilon\left(p^{\prime}\right)}}\right)^{\gamma-1}=1, \forall \gamma \in G_{F}$, as the characters $\psi_{\gamma}$ for $\gamma \in \Gamma_{0}$ generate the group $\left\{\psi_{\gamma}: \gamma \in G_{F}\right\}$. Therefore $\frac{a_{p^{\prime}}}{\sqrt{\epsilon\left(p^{\prime}\right)}} \in F^{*}$ and $v\left(a_{p^{\prime}}^{2} \epsilon^{-1}\left(p^{\prime}\right)\right) \equiv 0 \bmod 2$. Thus, both sides of equation (9) equal 1 . On the other hand, if $S_{p} \neq \emptyset$ and $\left(\frac{\tilde{t}_{\gamma_{0}}}{p}\right)=-1$, then one checks that $p^{\prime}$ is a candidate for the integer $n_{\gamma_{0}}$ defined in (6). Hence $\left(\frac{\tilde{t}_{\gamma_{0}}}{p}\right)^{f_{v} \cdot v\left(z_{n_{\gamma_{0}}}\right)}=(-1)^{f_{v} \cdot v\left(a_{p^{\prime}}^{2} \epsilon^{-1}\left(p^{\prime}\right)\right)}$, as desired.

So now assume that $S^{-}=\left\{t_{\gamma_{1}}=t_{1}, \cdots, t_{\gamma_{m}}=t_{m}\right\} \neq \emptyset$. By condition (b) on $\Gamma_{0}$, we have the first symbol $\left(\frac{\tilde{t}_{\gamma_{0}}}{v}\right)^{v\left(z_{n_{0}}\right)}=1$ in this case. Choose distinct primes $r_{j}$ with $a_{r_{j}} \neq 0$, for $j=0,1,2, \cdots, m-1$ recursively, satisfying the following properties:

(1) $r_{0}=p^{\prime}$,

(2) $\left(\frac{t_{\gamma}}{r_{j}}\right)=1$, for all $t_{\gamma} \in S^{+} \cup S_{p}$,

(3) $\left(\frac{t_{i}}{r_{j}}\right)=(-1)^{\delta_{i j}}\left(\frac{t_{i}}{r_{j-1}}\right)$, for all $i=1,2, \cdots, m$ (and $j \geq 1$ ).

Note that our choice of $r_{0}$ is consistent with property (2) above. Indeed, if $S_{p} \neq \emptyset$, then by condition $(\mathrm{b}),\left(\frac{t_{\gamma_{0}}}{p^{\prime}}\right) \stackrel{(*)}{=}\left(\frac{\tilde{t}_{\gamma_{0}}}{p}\right)=1$, and if $t_{\gamma} \in S^{+}$, then $\left(\frac{t_{\gamma}}{p^{\prime}}\right) \stackrel{(*)}{=}\left(\frac{t_{\gamma}}{p}\right)=1$.

Next we define

$$
n_{i}:= \begin{cases}r_{i-1} r_{i}, & \text { if } 1 \leq i \leq m-1, \\ r_{m-1}, & \text { if } i=m\end{cases}
$$

It can be checked that each $n_{i}$ satisfies the criterion given in (6) for $n_{\gamma_{i}}$. By the telescoping argument used in the proof of GGQ05, Thm. 4.3] or [BG03, Thm. 4.1.11], we get

$\bigotimes_{t_{\gamma} \in S \backslash S_{p}}\left(z_{n_{\gamma}}, t_{\gamma}\right)_{v}=\prod_{t_{\gamma} \in S^{-} \cup S^{+}}\left(\frac{t_{\gamma}}{p}\right)^{f_{v} \cdot v\left(z_{n_{\gamma}}\right)}=\prod_{i=1}^{m}(-1)^{f_{v} \cdot v\left(z_{n_{i}}\right)}=(-1)^{f_{v} \cdot v\left(a_{p^{\prime}}^{2} \epsilon^{-1}\left(p^{\prime}\right)\right)}$.

Applying equation (77) and Lemma [5.2] we get the following simplification of Theorem 5.1 .

$$
\left[X_{v}\right] \sim \epsilon_{p}(-1)^{\left[F_{v}: \mathbb{Q}_{p}\right]} \cdot\left(z_{n_{\gamma_{0}}}, p^{*}\right)_{v} \cdot(-1)^{f_{v} \cdot v\left(a_{p^{\prime}}^{2} \epsilon^{-1}\left(p^{\prime}\right)\right)},
$$

where, by equation (8), the middle symbol

$$
\left(z_{n_{\gamma_{0}}}, p^{*}\right)_{v}=(-1)^{v\left(z_{n_{0}}\right) e_{v}\left(p^{f v}-1\right) / 2} \cdot\left(\frac{\left(p^{*}\right)^{\prime}}{v}\right)^{v\left(z_{n_{\gamma_{0}}}\right)} \cdot\left(\frac{z_{n_{\gamma_{0}}}^{\prime}}{v}\right)^{e_{v}}
$$

when $S_{p}=\left\{t_{\gamma_{0}}\right\}$, and is taken to be trivial when $S_{p}=\emptyset$. The formulas (10) and (11) will be the starting point in our computation of the local Brauer class at a supercuspidal prime. Note that these formulas depend on the choice of $\Gamma_{0}$ satisfying conditions (a) and (b) stated before Lemma 5.2 


\section{UNRAMIFIED SUPERCUSPIDAL PRIMES}

In this section, we will prove Theorem 2.1 (a) in two parts, and then study some of its consequences.

Theorem 6.1. Let $p$ be an odd unramified supercuspidal prime with $\theta(s)+\theta(s)^{p} \neq$ 0 . Then, we have $\left[X_{v}\right] \sim(-1)^{f_{v} \cdot v\left(a_{p^{\prime}}^{2} \epsilon^{-1}\left(p^{\prime}\right)\right)}$, for $v \mid p$.

Proof. Let the set $\Gamma_{0}$ satisfy the two conditions before Lemma 5.2

Suppose $S_{p}=\left\{t_{\gamma_{0}}\right\} \neq \emptyset$; then by equation (10) it is enough to show that $\epsilon_{p}(-1)^{\left[F_{v}: \mathbb{Q}_{p}\right]} \cdot\left(z_{n_{\gamma_{0}}}, p^{*}\right)_{v}=1$. Let $g_{s} \in G_{p^{2}}$ be an element which is mapped to $s \in \mathbb{Q}_{p^{2}}^{*}$ under the reciprocity map. By local class field theory, we have $\psi\left(g_{s}\right)=$ $\psi\left(\left[N_{\mathbb{Q}_{p} \mid \mathbb{Q}_{p}}(s)\right]\right)$, for any Dirichlet (or idèlic) character $\psi$. Note that $p$ divides the conductor of a quadratic character $\psi$ if and only if $\psi$ is non-trivial on $(\mathbb{Z} / p \mathbb{Z})^{*}$. The norm $N_{\mathbb{Q}_{p^{2}} \mid \mathbb{Q}_{p}}(s)=s^{p+1}=x$, say, is a generator of the group $(\mathbb{Z} / p \mathbb{Z})^{*}$ inside $\mathbb{Z}_{p}^{*}$. Hence for any $\gamma \in \Gamma_{0}, \psi_{\gamma}\left(g_{s}\right)=-1 \Longleftrightarrow p^{*} \mid t_{\gamma} \Longleftrightarrow \gamma=\gamma_{0}$, where the last implication follows from condition (a) on $\Gamma_{0}$. Looking at the definition of $n_{\gamma_{0}}$ given in (6), we conclude that $\forall \gamma \in \Gamma_{0}$ and hence $\forall \gamma \in G_{F}, \psi_{\gamma}\left(g_{s}\right)=\psi_{\gamma}\left(n_{\gamma_{0}}\right)$. Hence it follows from (11) and (5) that $\left(\frac{\alpha\left(g_{s}\right)}{\sqrt{\epsilon\left(g_{s}\right)}}\right)^{\gamma-1}=\left(\frac{a_{n_{\gamma_{0}}}}{\sqrt{\epsilon\left(n_{\gamma_{0}}\right)}}\right)^{\gamma-1}$, for all $\gamma \in G_{F}$. So we have

$$
z_{n_{\gamma_{0}}}=\frac{a_{n_{\gamma_{0}}}^{2}}{\epsilon\left(n_{\gamma_{0}}\right)} \equiv \frac{\alpha^{2}\left(g_{s}\right)}{\epsilon\left(g_{s}\right)} \equiv \frac{\left(\theta(s)+\theta(s)^{p}\right)^{2}}{\epsilon_{p}^{-1}(x)} \bmod F^{* 2},
$$

where the last congruence is by part (2) of Proposition 3.1, noting that the trace $\theta(s)+\theta(s)^{p}$ is non-zero by assumption. As $\theta(s)+\theta(s)^{p}=\operatorname{Tr}_{\mathbb{Q}_{p} 2} \mid \mathbb{Q}_{p}(\theta(s)) \in \mathbb{Q}_{p}^{*} \subseteq F_{v}^{*}$, we get $z_{n_{\gamma_{0}}} \equiv \epsilon_{p}(x) \bmod \left(F_{v}^{*}\right)^{2}$, and $v\left(z_{n_{\gamma_{0}}}\right) \equiv 0 \bmod 2$. By equation (11), we get $\left(z_{n_{\gamma_{0}}}, p^{*}\right)_{v}=\left(\frac{z_{n_{\gamma_{0}}}^{\prime}}{v}\right)^{e_{v}}=\left(\frac{\epsilon_{p}(x)}{v}\right)^{e_{v}}=\left(\frac{\epsilon_{p}(x)}{p}\right)^{f_{v} e_{v}}=\left(\epsilon_{p}(x)^{(p-1) / 2}\right)^{e_{v} f_{v}}=$ $\epsilon_{p}(-1)^{e_{v} f_{v}}$; therefore $\epsilon_{p}(-1)^{\left[F_{v}: \mathbb{Q}_{p}\right]} \cdot\left(z_{n_{\gamma_{0}}}, p^{*}\right)_{v}=\left(\epsilon_{p}(-1)^{e_{v} f_{v}}\right)^{2}=1$.

If $S_{p}=\emptyset$, then $\psi_{\gamma}\left(g_{s}\right)=1$ for all $\gamma \in G_{F}$, and a similar argument shows that $\left(\theta(s)+\theta(s)^{p}\right)^{2} \equiv \epsilon_{p}^{-1}(x) \bmod F^{* 2}$. Thus we get $\epsilon_{p}(x) \in F_{v}^{* 2}$, which implies that $\left(\frac{\epsilon_{p}(x)}{v}\right)=\left(\frac{\epsilon_{p}(x)}{p}\right)^{f_{v}}=\epsilon_{p}(-1)^{f_{v}}=1$. Hence $\epsilon_{p}(-1)^{\left[F_{v}: \mathbb{Q}_{p}\right]}=1$, and the result follows by equation (10).

Note that if $p$ is a supercuspidal prime of level zero, then by the regularity condition (1) on $\theta$ in the definition of an admissible pair, we have $\theta(s)^{p-1} \neq 1$. The condition on $\theta(s)$ in the hypothesis of Theorem 6.1 is equivalent to $\theta(s)^{p-1} \neq-1$. However, for an unramified supercuspidal prime of positive level we remove this condition now.

Theorem 6.2. Let $p$ be an odd unramified supercuspidal prime of level $>0$ for a $p$-minimal non-CM newform $f$. Then, we have $\left[X_{v}\right] \sim(-1)^{f_{v} \cdot v\left(a_{p^{\prime}}^{2} \epsilon^{-1}\left(p^{\prime}\right)\right)}$, for $v \mid p$.

Proof. Since $p$ is an unramified supercuspidal prime of level $>0, N_{p} \geq 4$. We twist $f$ by a suitable character at $p$ if necessary, and assume that $\epsilon_{p}$ is tame. Note that the formula to be proved is invariant under twist by a character of the $p$-power conductor. As $f$ was $p$-minimal to begin with, $N_{p}$ cannot decrease by twisting. 
Thus the hypothesis of Lemma 4.1 is satisfied. If $\theta(s)+\theta(s)^{p} \neq 0$, we are done by

Theorem 6.1 So assume $\theta(s)+\theta(s)^{p}=0$, i.e., $\theta(s)^{p-1}=-1$.

As explained in the proof of Theorem 6.1, if $S_{p} \neq \emptyset$, then

$$
z_{n_{\gamma_{0}}}=\frac{a_{n_{\gamma_{0}}}^{2}}{\epsilon\left(n_{\gamma_{0}}\right)} \equiv \frac{\alpha^{2}\left(g_{s}\right)}{\epsilon\left(g_{s}\right)} \bmod \left(F^{*}\right)^{2} .
$$

Note that $\theta^{\sigma}(s)=\theta(s)^{p}=-\theta(s)$. By Lemma 4.1 $\exists \tau \in I_{w}(K)$ such that

$$
\alpha\left(g_{s}\right) \equiv \alpha\left(g_{s} \tau\right) \equiv\left(\theta+\theta^{\sigma}\right)\left(g_{s} \tau\right) \equiv \theta(s)\left(\zeta_{p}-\zeta_{p}^{-1}\right) \quad \bmod F^{*} .
$$

Using equations (3) and (4), we get

$$
\epsilon\left(g_{s}\right)=\epsilon\left(\left[N_{\mathbb{Q}_{p^{2}} \mid \mathbb{Q}_{p}}(s)\right]\right)=\epsilon_{p}^{-1}\left(s^{p+1}\right)=\theta\left(s^{p+1}\right) .
$$

By equations (12), (13), and (14) and using the fact that $\theta(s)^{p-1}=-1$, we get

$$
z_{n_{\gamma_{0}}} \equiv-\left(\zeta_{p}-\zeta_{p}^{-1}\right)^{2} \equiv-p^{2}\left(\zeta_{p}-\zeta_{p}^{-1}\right)^{2} \bmod \left(F^{*}\right)^{2} .
$$

Suppose $p \equiv 3 \bmod 4$; then $\sqrt{-p}\left(\zeta_{p}-\zeta_{p}^{-1}\right)$ is a totally real element in $\mathbb{Q}\left(\zeta_{p}\right)$, and hence it is contained in the field $\mathbb{Q}\left(\zeta_{p}+\zeta_{p}^{-1}\right) \subseteq F$, by Lemma 4.1. Thus we have $-p\left(\zeta_{p}-\zeta_{p}^{-1}\right)^{2} \in\left(F^{*}\right)^{2}$, and hence

$$
\left(\frac{z_{n_{\gamma_{0}}}^{\prime}}{v}\right)=\left(\frac{\left(-p^{2}\left(\zeta_{p}-\zeta_{p}^{-1}\right)^{2}\right)^{\prime}}{v}\right)=\left(\frac{(p)^{\prime}}{v}\right),
$$

where we write $(p)^{\prime}$ for the prime-to- $\pi_{v}$ part of $p$ to distinguish it from the auxiliary prime $p^{\prime}$. From equation (15), we have the valuation

$$
v\left(z_{n_{\gamma_{0}}}\right) \equiv v\left(-\left(\zeta_{p}-\zeta_{p}^{-1}\right)^{2}\right)=2 e_{v} /(p-1) \equiv e_{v} \bmod 2 .
$$

As $p \equiv 3 \bmod 4,(N v-1) / 2=\left(p^{f_{v}}-1\right) / 2 \equiv f_{v} \bmod 2$. Hence by (11) and (16), $\left(z_{n_{\gamma_{0}}}, p^{*}\right)_{v}=(-1)^{e_{v} e_{v} f_{v}} \cdot\left(\frac{(-p)^{\prime}}{v}\right)^{e_{v}} \cdot\left(\frac{(p)^{\prime}}{v}\right)^{e_{v}}=(-1)^{e_{v} f_{v}} \cdot\left(\frac{-1}{v}\right)^{e_{v}}=(-1)^{e_{v} f_{v}}$. $\left(\frac{-1}{p}\right)^{e_{v} f_{v}}=1$. By (44), $\epsilon_{p}(-1)=\theta(-1)=\theta(s)^{\left(p^{2}-1\right) / 2}=\left(\theta(s)^{p-1}\right)^{(p+1) / 2}=$ $(-1)^{(p+1) / 2}=1$, and therefore by (10) , we get $\left[X_{v}\right] \sim(-1)^{f_{v} \cdot v\left(a_{p^{\prime}}^{2} \epsilon^{-1}\left(p^{\prime}\right)\right)}$.

Now suppose $p \equiv 1 \bmod 4$. By Lemma 4.1, we get that $e_{v}$ is even, and $\sqrt{p}=\sqrt{p^{*}} \in \mathbb{Q}_{p}\left(\zeta_{p}+\zeta_{p}^{-1}\right)^{*} \subseteq F_{v}^{*}$; hence $\left(\frac{\left(p^{*}\right)^{\prime}}{v}\right)=1$. As all the other symbols involved in $\epsilon_{p}(-1)^{\left[F_{v}: \mathbb{Q}_{p}\right]} \cdot\left(z_{n_{\gamma_{0}}}, p^{*}\right)_{v}$ have an $e_{v}$ in the exponent, we get $\left[X_{v}\right] \sim(-1)^{f_{v} \cdot v\left(a_{p^{\prime}}^{2} \epsilon^{-1}\left(p^{\prime}\right)\right)}$, by equation (10).

If $S_{p}=\emptyset$, then the result follows trivially from the fact that $\epsilon_{p}(-1)^{\left[F_{v}: \mathbb{Q}_{p}\right]}=1$ in all cases, as shown above.

Remark. The result above is false in general for supercuspidal primes of level zero. There exist $p$-minimal newforms with $C_{p}<N_{p}=2$, with $\theta(s)+\theta(s)^{p}=0$, such that $f_{v}$ is even but $X_{v}$ is ramified at some $v \mid p$; see example (5) in the last section.

Theorems 6.1 and 6.2 imply Theorem 2.1 (a). Indeed, if $\theta(s)+\theta(s)^{p} \neq 0$, the first theorem applies. Otherwise, $\theta(s)+\theta(s)^{p}$ vanishes. By the hypothesis made in Theorem 2.1 we may assume that the $p$-minimal twist of $f$ has a positive level, and the second theorem applies. We remark here that the vanishing of $\theta(s)+\theta(s)^{p}$ does not depend on the twist.

Corollary 6.3. Let $p$ be an odd unramified supercuspidal prime for $f$. Also assume that $\theta(s)+\theta(s)^{p} \neq 0$, if the p-minimal twist of $f$ is of level 0 . 
(a) If $N^{\prime}=1$, or if $p$ has odd order in $\left(\mathbb{Z} / N^{\prime} \mathbb{Z}\right)^{*}$, then $X_{v}$ is a matrix algebra over $F_{v}$.

(b) If $\mathbb{Q}_{p^{2}} \subseteq F_{v}$, then $X_{v}$ is a matrix algebra over $F_{v}$.

(c) If $X_{v}$ has non-trivial Brauer class in $\operatorname{Br}\left(F_{v}\right)$, then $v$ must divide $\operatorname{disc}(E \mid F)$.

Proof. We have:

(a) For any Dirichlet character $\chi$, let $\chi^{\prime}$ denote its prime-to- $p$ component.

If $N^{\prime}=1$, then $\chi_{\gamma}\left(p^{\prime}\right) \stackrel{(*)}{=} \chi_{\gamma}^{\prime}\left(p^{\prime}\right)=1$, for all $\gamma \in \Gamma$. Hence $a_{p^{\prime}} \in F^{*}$.

If $N^{\prime} \neq 1$, let $n=2 m+1$ be the order of $p \bmod N^{\prime}$, i.e., $p^{n} \equiv 1$ $\bmod N^{\prime}$. Hence we have $\chi_{\gamma}\left(p^{\prime}\right)^{n}=\chi_{\gamma}^{\prime}\left(p^{n}\right)=1$, for all $\gamma \in \Gamma$. We know that $\chi_{\gamma}^{\prime}(p)^{2}=\chi_{\gamma}\left(p^{\prime}\right)^{2}=\epsilon\left(p^{\prime}\right)^{\gamma-1}$, for all $\gamma \in \Gamma$. Hence $\chi_{\gamma}\left(p^{\prime}\right)=\chi_{\gamma}^{\prime}(p)^{n-2 m}=$ $\left(\chi_{\gamma}^{\prime}(p)^{2}\right)^{-m}=\left(\epsilon\left(p^{\prime}\right)^{\gamma-1}\right)^{-m}$, which implies that $a_{p^{\prime}}^{\gamma-1}=\left(\epsilon\left(p^{\prime}\right)^{-m}\right)^{\gamma-1}$, for all $\gamma \in \Gamma$. So $a_{p^{\prime}} \equiv \epsilon\left(p^{\prime}\right)^{-m} \bmod F^{*}$.

Now use Theorems 6.1 and 6.2 to get the result in both cases.

(b) $\mathbb{Q}_{p^{2}} \subseteq F_{v}$ would imply that $f_{v}$ is even. Now apply Theorems 6.1 and 6.2. Note that here $K=\mathbb{Q}_{p^{2}}$, so we have proved a part of Corollary 2.3.

(c) By Theorems 6.1 and 6.2. $\left[X_{v}\right] \sim-1$ implies that $v\left(a_{p^{\prime}}^{2} \epsilon^{-1}\left(p^{\prime}\right)\right)$ is odd. If $v$ is extended to a valuation on $E^{*}$, then clearly $v\left(a_{p^{\prime}}\right)$ is not an integer. Hence $v$ ramifies in $E$, or $v \mid \operatorname{disc}(E \mid F)$.

\section{RAMIFIED SUPERCUSPIDAL PRIMES}

In this section we will prove parts (b) and (c) of Theorem 2.1] and derive some consequences.

Let $p$ be an odd ramified supercuspidal prime for $f$. As $K \mid \mathbb{Q}_{p}$ is ramified, there are two possible choices for $K$. If $\left(p, K \mid \mathbb{Q}_{p}\right)=1$, then $K=\mathbb{Q}_{p}(\sqrt{-p})$, and if $\left(p, K \mid \mathbb{Q}_{p}\right)=-1$, then $K=\mathbb{Q}_{p}\left(\sqrt{-p \zeta_{p-1}}\right)$. Depending on $K$, we can always choose a uniformizer $\pi=\sqrt{-p}$ or $\sqrt{-p \zeta_{p-1}}$, and write $K=\mathbb{Q}_{p}(\pi)$. For $\sigma \in G_{p}$, any lift of the generator of $\operatorname{Gal}\left(K \mid \mathbb{Q}_{p}\right)$, we have $\pi^{\sigma}=-\pi$, and $N_{K \mid \mathbb{Q}_{p}}(\pi)=-\pi^{2}$.

Let $p^{\prime}$ and $p^{\prime \prime}$ be as in Section 2, satisfying properties $(*)$ and $(* *)$ respectively.

Theorem 7.1. Let $p \equiv 1 \bmod 4$ be a ramified supercuspidal prime. Then, for any prime $v \mid p$ in $F$, the Brauer class is given by $\left[X_{v}\right] \sim(-1)^{f_{v} \cdot v\left(a_{p^{\prime}}^{2} \epsilon^{-1}\left(p^{\prime}\right)\right)}$.

Proof. The formula to be proved is invariant under twist by a Dirichlet character of the $p$-power conductor. So we twist $f$ by a suitable character, if necessary, and then assume that $f$ is $p$-minimal. Since $p$ is a ramified supercuspidal prime, even after applying the twist the level will still be positive, i.e., the hypothesis of Lemma 4.2 is satisfied.

Since $p \equiv 1 \bmod 4, e_{v}$ is even and $\sqrt{p} \in \mathbb{Q}\left(\zeta_{p}+\zeta_{p}^{-1}\right)^{*} \subseteq F^{*}$, by Lemma 4.2 . As $p^{*}=p$ is a square in $F_{v}^{*}$, we get $\left(\frac{\left(p^{*}\right)^{\prime}}{v}\right)=1$. Hence by (10) and (11), we get $\left[X_{v}\right] \sim(-1)^{f_{v} \cdot v\left(a_{p^{\prime}}^{2} \epsilon^{-1}\left(p^{\prime}\right)\right)}$.

Remark. Since the formula turns out to be the same as in the case of unramified supercuspidal primes, Corollary 6.3 also holds for ramified supercuspidal primes $p \equiv 1 \bmod 4$, as stated in Corollary 2.2. But note that none of the results in Corollary 2.2 are true for ramified supercuspidal primes $p \equiv 3 \bmod 4$; see the examples in the last section. 
Lemma 7.2. For any odd ramified supercuspidal prime $p$, the valuations of $a_{p^{\prime}}$ and $a_{p^{\prime \prime}}$ are related by the following equation:

$$
(-1)^{v\left(a_{p^{\prime}}^{2} \epsilon^{-1}\left(p^{\prime}\right)\right)}=(-1)^{e_{v}(k-1)} \cdot\left(\left(\frac{-1}{p}\right) \epsilon_{p}(-1)\right)^{2 e_{v} /(p-1)} \cdot\left(p, K \mid \mathbb{Q}_{p}\right)^{v\left(a_{p^{\prime \prime}}^{2} \epsilon^{-1}\left(p^{\prime \prime}\right)\right)} .
$$

Proof. The equation to be proved is invariant under twist by a character of the $p$ power conductor. So without loss of generality we assume $\epsilon_{p}$ to be tame, so that we can apply Lemma4.1. We write the ramified quadratic extension $K$ as $K=\mathbb{Q}_{p}(\pi)$, where either $\pi=\sqrt{-p}$ or $\pi=\sqrt{-p \zeta_{p-1}}$.

Let $g_{\pi} \in G_{K}$ be an element whose image under the reciprocity map is $\pi \in K^{*}$. Taking the determinant of $\rho_{f}$ (described in Section 4) at $g_{\pi}$, we get

$$
\begin{gathered}
\theta(\pi) \theta^{\sigma}(\pi)=\theta(\pi) \theta(-\pi)=\chi_{\ell}^{k-1}\left(g_{\pi}\right) \epsilon\left(g_{\pi}\right) \\
\Longrightarrow \theta(\pi)^{2} \epsilon^{-1}\left(g_{\pi}\right)=\theta(-1) p^{k-1} .
\end{gathered}
$$

If $\theta(-1)=1$, then $\alpha\left(g_{\pi}\right) \equiv\left(\theta+\theta^{\sigma}\right)(\pi)=2 \theta(\pi) \equiv \theta(\pi) \bmod F^{*}$. So by (18), we have $\alpha^{2}\left(g_{\pi}\right) \epsilon^{-1}\left(g_{\pi}\right) \equiv p^{k-1} \bmod \left(F^{*}\right)^{2}$. If $\theta(-1)=-1$, we use the element $\tau \in$ $I_{w}(K)$ given by Lemma 4.1 to get $\alpha\left(g_{\pi}\right) \equiv \alpha\left(g_{\pi} \tau\right) \equiv\left(\theta+\theta^{\sigma}\right)\left(g_{\pi} \tau\right) \equiv \theta(\pi)\left(\zeta_{p}-\zeta_{p}^{-1}\right)$ $\bmod F^{*}$, and by (18), we have $\alpha^{2}\left(g_{\pi}\right) \epsilon^{-1}\left(g_{\pi}\right) \equiv-p^{k-1}\left(\zeta_{p}-\zeta_{p}^{-1}\right)^{2} \bmod \left(F^{*}\right)^{2}$. Therefore using $v(p)=e_{v}$ and $v\left(\left(\zeta_{p}-\zeta_{p}^{-1}\right)^{2}\right)=2 e_{v} /(p-1)$, we get

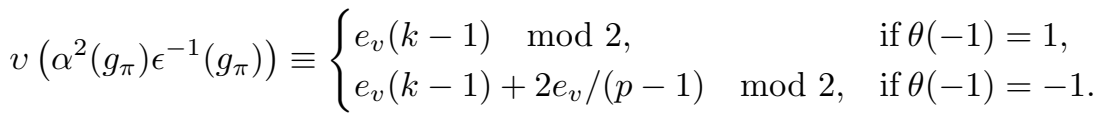

By (44), $\theta(-1)=\left(\frac{-1}{p}\right) \epsilon_{p}(-1)$, so the congruence above can be written as

$$
(-1)^{v\left(\alpha^{2}\left(g_{\pi}\right) \epsilon^{-1}\left(g_{\pi}\right)\right)}=(-1)^{e_{v}(k-1)} \cdot\left(\left(\frac{-1}{p}\right) \epsilon_{p}(-1)\right)^{2 e_{v} /(p-1)} .
$$

By class field theory, $g_{\pi} \in G_{p} \subseteq G_{\mathbb{Q}}$ is mapped to $\left[N_{K \mid \mathbb{Q}_{p}}(\pi)\right] \in \mathbb{Q}_{p}^{*} \subseteq \mathbb{A}_{\mathbb{Q}}^{*}$. Each $\chi_{\gamma}$ is realized as an idèlic character as in equation (3), and hence as a Galois character.

If $\left(p, K \mid \mathbb{Q}_{p}\right)=1$, then $N_{K \mid \mathbb{Q}_{p}}(\pi)=p$, and for all $\gamma \in \Gamma$, we have $\chi_{\gamma}\left(g_{\pi}\right)=$ $\chi_{\gamma}([p])=\chi_{\gamma}^{\prime}(p) \stackrel{(*)}{=} \chi_{\gamma}\left(p^{\prime}\right)$. Hence applying (1) and (3), we get that

$$
\alpha^{2}\left(g_{\pi}\right) \epsilon^{-1}\left(g_{\pi}\right) \equiv a_{p^{\prime}}^{2} \epsilon^{-1}\left(p^{\prime}\right) \bmod \left(F^{*}\right)^{2} .
$$

If $\left(p, K \mid \mathbb{Q}_{p}\right)=-1$, then $N_{K \mid \mathbb{Q}_{p}}(\pi)=p \zeta_{p-1}$. So for all $\gamma \in \Gamma$, we have $\chi_{\gamma}\left(g_{\pi}\right)=$ $\chi_{\gamma}\left(\left[p \zeta_{p-1}\right]\right) \stackrel{(* *)}{=} \chi_{\gamma}^{\prime}(p) \chi_{\gamma, p}^{-1}\left(p^{\prime \prime}\right) \stackrel{(*)}{=} \chi_{\gamma}\left(p^{\prime}\right) \chi_{\gamma}^{-1}\left(p^{\prime \prime}\right)$. Applying (10) and (푸), we get

$$
\left(\alpha^{2}\left(g_{\pi}\right) \epsilon^{-1}\left(g_{\pi}\right)\right) \cdot\left(a_{p^{\prime \prime}}^{2} \epsilon^{-1}\left(p^{\prime \prime}\right)\right) \equiv a_{p^{\prime}}^{2} \epsilon^{-1}\left(p^{\prime}\right) \bmod \left(F^{*}\right)^{2} .
$$

Now the result follows from equation (19) above.

Corollary 7.3. Let $p \equiv 1 \bmod 4$ be a ramified supercuspidal prime such that $K=\mathbb{Q}_{p}(\sqrt{p})$. Then, for any prime $v \mid p$ in $F$, we have $\left[X_{v}\right] \sim \epsilon_{p}(-1)^{2\left[F_{v}: \mathbb{Q}_{p}\right] /(p-1)}$.

Proof. As explained in the proof of Theorem 7.1, without loss of generality we may assume that $f$ is $p$-minimal, where $p$ is a supercuspidal prime of level $>0$. By the hypothesis $K=\mathbb{Q}_{p}(\sqrt{p})=\mathbb{Q}_{p}(\sqrt{-p})$, as $p \equiv 1 \bmod 4$. Hence we have $\left(\frac{-1}{p}\right)=1=\left(p, K \mid \mathbb{Q}_{p}\right)$. By Lemma 4.2, $e_{v}$ is even. Now we apply Lemma 7.2 to the statement of Theorem 7.1 to get the result. 
Theorem 7.4. Let $p \equiv 3 \bmod 4$ be a ramified supercuspidal prime. Let $v$ be a prime in $F$ such that $e_{v}$ is odd. Then $\left[X_{v}\right] \sim\left((-1)^{k} a_{p^{\prime \prime}}^{2} \epsilon^{-1}\left(p^{\prime \prime}\right), K F_{v} \mid F_{v}\right)$.

Proof. Since $e_{v}$ is odd, $K \nsubseteq F_{v}$ and $K F_{v} \mid F_{v}$ is a ramified proper quadratic extension. So we can and do choose a uniformizer $\pi_{v} \in N_{K F_{v} \mid F_{v}}\left(K F_{v}\right)^{*} \subseteq F_{v}^{*}$. Note that in this case, we have $N_{K F_{v} \mid F_{v}}\left(\mathcal{O}_{K F_{v}}^{*}\right)=\mathcal{O}_{F_{v}}^{*^{2}}$, where $\mathcal{O}^{*}$ denotes units. Hence for any $a=\pi_{v}^{v(a)} \cdot a^{\prime} \in F_{v}^{*}$, we have $\left(\frac{a^{\prime}}{v}\right)=\left(a, K F_{v} \mid F_{v}\right)$.

If $\left(p, K \mid \mathbb{Q}_{p}\right)=1$, then $\left(\frac{(p)^{\prime}}{v}\right)=\left(p, K F_{v} \mid F_{v}\right)=1$. If $\left(p, K \mid \mathbb{Q}_{p}\right)=-1$, then $K=$ $\mathbb{Q}_{p}\left(\sqrt{-p \zeta_{p-1}}\right)$ and $N_{K F_{v} \mid F_{v}}\left(\sqrt{-p \zeta_{p-1}}\right)=p \zeta_{p-1}$, so $\left(\frac{\left(p \zeta_{p-1}\right)^{\prime}}{v}\right)=\left(p \zeta_{p-1}, K F_{v} \mid F_{v}\right)$ $=1$, which implies that $\left(\frac{(p)^{\prime}}{v}\right)=\left(\frac{\zeta_{p-1}}{v}\right)=(-1)^{f_{v}}$. Combining both the cases we get

$$
\left(\frac{\left(p^{*}\right)^{\prime}}{v}\right)=\left(\frac{-1}{v}\right)\left(\frac{(p)^{\prime}}{v}\right)=\left(\frac{-1}{p}\right)^{f_{v}}\left(\left(p, K \mid \mathbb{Q}_{p}\right)\right)^{f_{v}}=\left(-\left(p, K \mid \mathbb{Q}_{p}\right)\right)^{f_{v}} .
$$

If $\emptyset \neq S_{p}=\left\{t_{\gamma_{0}}\right\}$, then as in the proof of Theorem 6.1, we use (6) and (**) to choose $n_{\gamma_{0}}$ to be $p^{\prime \prime}$; hence $z_{n_{\gamma_{0}}}=a_{p^{\prime \prime}}^{2} \epsilon^{-1}\left(p^{\prime \prime}\right)$. Now using (10), (11), (20), Lemma 7.2. and the facts that $e_{v}$ is odd, $(p-1) / 2$ is odd and so $\left(p^{f_{v}}-1\right) / 2 \equiv f_{v} \bmod 2$, we get

$$
\begin{aligned}
& {\left[X_{v}\right] \stackrel{[10]}{\sim} \quad \epsilon_{p}(-1)^{\left[F_{v}: \mathbb{Q}_{p}\right]} \cdot\left(z_{n_{\gamma_{0}}}, p^{*}\right)_{v} \cdot(-1)^{f} \cdot v\left(a_{p^{\prime}}^{2} \epsilon^{-1}\left(p^{\prime}\right)\right) } \\
& \epsilon_{p}(-1)^{e_{v} f_{v}} \cdot(-1)^{v\left(z_{n_{\gamma_{0}}}\right) \cdot e_{v} \cdot\left(p^{f} f_{v}-1\right) / 2}\left(\frac{\left(p^{*}\right)^{\prime}}{v}\right)^{v\left(z_{n_{0}}\right)}\left(\frac{\left(z_{n_{\gamma_{0}}}\right)^{\prime}}{v}\right)^{e_{v}} \\
& \cdot(-1)^{f_{v} \cdot v\left(a_{p^{\prime}}^{2} \epsilon^{-1}\left(p^{\prime}\right)\right)} \\
& \cdot\left((-1)^{(k-1)}\left(-\epsilon_{p}(-1)\right)\left(p, K \mid \mathbb{Q}_{p}\right)^{v\left(a_{p^{\prime \prime}}^{2} \epsilon^{-1}\left(p^{\prime \prime}\right)\right)}\right)^{f_{v}} \\
&=(-1)^{k f_{v}} \cdot\left(\frac{\left(z_{n_{\gamma_{0}}}\right)^{\prime}}{v}\right) \sim\left(\frac{\left((-1)^{k} z_{n_{\gamma_{0}}}\right)^{\prime}}{v}\right) \\
&=\left((-1)^{k} z_{n_{\gamma_{0}}}, K F_{v} \mid F_{v}\right) \\
&=\left((-1)^{k} a_{p^{\prime \prime}}^{2} \epsilon^{-1}\left(p^{\prime \prime}\right), K F_{v} \mid F_{v}\right) .
\end{aligned}
$$

If $S_{p}=\emptyset$, then $\psi_{\gamma}\left(p^{\prime \prime}\right)=1, \forall \gamma \in G_{F}$, so it follows from (5) that $a_{p^{\prime \prime}}^{2} \epsilon^{-1}\left(p^{\prime \prime}\right) \in$ $F^{* 2} \subseteq F_{v}^{* 2}$. Therefore the symbol $\left(z_{n_{\gamma_{0}}}, p^{*}\right)_{v}$ in equation (10) can be replaced by the trivial symbol $\left(a_{p^{\prime \prime}}^{2} \epsilon^{-1}\left(p^{\prime \prime}\right), p^{*}\right)_{v}$, and then the same proof (as above) works.

The next lemma is a basic application of algebraic number theory and hence we state it without proof.

Lemma 7.5. Let $\varpi$ be any uniformizer in $\mathbb{Z}_{p}$. If $e_{v}$ is even, then either $\sqrt{\varpi} \in F_{v}^{*}$ or $\sqrt{\varpi \zeta_{p^{f_{v}-1}}} \in F_{v}^{*}$. Moreover, if $K \mid \mathbb{Q}_{p}$ is a ramified quadratic extension, then $K F_{v} \mid F_{v}$ is an unramified extension of degree 1 or 2 .

Theorem 7.6. Let $p \equiv 3 \bmod 4$ be a ramified supercuspidal prime and suppose that $e_{v}$ is even. Then, the formula $\left[X_{v}\right] \sim\left((-1)^{k} a_{p^{\prime \prime}}^{2} \epsilon^{-1}\left(p^{\prime \prime}\right), K F_{v} \mid F_{v}\right)$ still holds. 
Proof. As $p^{\prime \prime}$ is a candidate for the integer $n_{\gamma_{0}}$, by (10), (11), Lemma 7.2 and the assumption that $e_{v}$ is even, we get that if $S_{p} \neq \emptyset$, then

$$
\begin{aligned}
{\left[X_{v}\right] } & \sim\left(\frac{\left(p^{*}\right)^{\prime}}{v}\right)^{v\left(a_{p^{\prime \prime}}^{2} \epsilon^{-1}\left(p^{\prime \prime}\right)\right)} \cdot(-1)^{f_{v} \cdot v\left(a_{p^{\prime}}^{2} \epsilon^{-1}\left(p^{\prime}\right)\right)} \\
& \sim\left(\left(\frac{(-p)^{\prime}}{v}\right) \cdot\left(p, K \mid \mathbb{Q}_{p}\right)^{f_{v}}\right)^{v\left(a_{p^{\prime \prime}}^{2} \epsilon^{-1}\left(p^{\prime \prime}\right)\right)} .
\end{aligned}
$$

So we compute the symbol $\left(\frac{(-p)^{\prime}}{v}\right)$ case by case. We use the fact that since $p \equiv 3$ $\bmod 4$, the possibilities for $K$ are $\mathbb{Q}_{p}(\sqrt{-p})$ and $\mathbb{Q}_{p}(\sqrt{p})=\mathbb{Q}_{p}\left(\sqrt{-p \zeta_{p-1}}\right)$, and these occur exactly when $\left(p, K \mid \mathbb{Q}_{p}\right)=1$ or -1 , respectively.

Case 1. Assume $K \subseteq F_{v}$. If $\left(p, K \mid \mathbb{Q}_{p}\right)=1$, then $\sqrt{-p} \in K \subseteq F_{v} \Rightarrow\left(\frac{(-p)^{\prime}}{v}\right)=1$. If $\left(p, K \mid \mathbb{Q}_{p}\right)=-1$, then $\sqrt{p} \in K \subseteq F_{v} \Rightarrow\left(\frac{(p)^{\prime}}{v}\right)=1 \Rightarrow\left(\frac{(-p)^{\prime}}{v}\right)=\left(\frac{-1}{v}\right)=(-1)^{f_{v}}$.

Case 2. Assume $K \nsubseteq F_{v}$. By Lemma 7.5 $K F_{v} \mid F_{v}$ is a proper quadratic unramified extension. If $\left(p, K \mid \mathbb{Q}_{p}\right)=1$, then $\sqrt{-p} \notin F_{v}$, so by Lemma 7.5 with $\varpi=-p$,

$$
\sqrt{-p \zeta_{p^{f_{v}-1}}} \in F_{v} \Rightarrow\left(\frac{\left(-p \zeta_{p^{f_{v}-1}}\right)^{\prime}}{v}\right)=1 \Rightarrow\left(\frac{(-p)^{\prime}}{v}\right)=\left(\frac{\zeta_{p^{f_{v}-1}}}{v}\right)=-1 .
$$

If $\left(p, K \mid \mathbb{Q}_{p}\right)=-1$, then $\sqrt{p} \notin F_{v}$, so by Lemma 7.5 with $\varpi=p$,

$$
\sqrt{p \zeta_{p^{f v}-1}} \in F_{v} \Rightarrow\left(\frac{\left(p \zeta_{p^{f v}-1}\right)^{\prime}}{v}\right)=1 \Rightarrow\left(\frac{(-p)^{\prime}}{v}\right)=\left(\frac{-\zeta_{p^{f v}-1}}{v}\right)=(-1)^{f_{v}+1} \text {. }
$$

Applying these to equation (21), we get

$$
\left[X_{v}\right] \sim \begin{cases}1, & \text { if } K \subseteq F_{v} \\ (-1)^{v\left(a_{p^{\prime \prime}}^{2} \epsilon^{-1}\left(p^{\prime \prime}\right)\right)}, & \text { otherwise. }\end{cases}
$$

It can be checked that this formula is valid even if $S_{p}=\emptyset$, as in the proof of Theorem 7.4. Equivalently, $\left[X_{v}\right] \sim\left((-1)^{k} a_{p^{\prime \prime}}^{2} \epsilon^{-1}\left(p^{\prime \prime}\right), K F_{v} \mid F_{v}\right)$, as $K F_{v} \mid F_{v}$ is unramified.

Remark. Note that the factor $(-1)^{k}$ above does not have any significance, since $K F_{v} \mid F_{v}$ is unramified. We keep it only to get a uniform formula for all $e_{v}$, even or odd.

\section{NUMERICAL EXAMPLES}

Here are some numerical examples in support of our results. We used the program Sage to compute the admissible pair $(K, \theta)$ attached to a supercuspidal prime of a newform in some cases. One may also directly check the hypothesis on $\theta(s)$ in Theorem 6.1 in the case of level zero supercuspidals, using equation (4), together with the fact that the order of $\theta(s)$ divides $p^{2}-1$, but not $p-1$. To determine the local Brauer class $\left[X_{v}\right]$, we used the program Endohecke for newforms with quadratic character, and the data from the tables in GGQ05 and Qu05 for newforms with arbitrary character. 
(1) $f \in S_{5}(75,[1,0]), E=\mathbb{Q}(\sqrt{-35}), F=\mathbb{Q} ; p=5$ is an unramified supercuspidal prime of level zero with $\theta(s)+\theta(s)^{5} \neq 0$. For $p=5, p^{\prime}=101$ satisfies $(*)$ and we computed $a_{101}^{2}=-1800^{2} \cdot 35$. So $f_{v} \cdot v\left(a_{p^{\prime}}^{2} \epsilon^{-1}\left(p^{\prime}\right)\right)=$ $1 \cdot v_{5}\left(1800^{2} \cdot 35\right)=5 \equiv 1 \bmod 2$. By Theorem 6.1 $X_{5}$ is ramified.

There exists another newform, say $g \in S_{5}(75,[1,0])$, with $F=\mathbb{Q}$ and $E=\mathbb{Q}(\sqrt{-14})$, such that $p=5$ is an unramified supercuspidal prime of level zero with $\theta(s)+\theta(s)^{5} \neq 0$. By Corollary 6.3 (c), $X_{5}$ has to be unramified. But, the admissible pairs attached to $f$ and $g$ at $p$ are equivalent. This shows that the $\overline{\mathbb{Q}}_{\ell}$-isomorphism class of the local $\ell$-adic Galois representation at a supercuspidal prime $p$ fails to predict the Brauer class $\left[X_{v}\right]$ above $p$, even in the simplest case $F=\mathbb{Q}$.

(2) $f \in S_{2}(72,[1,1,3]), E=\mathbb{Q}(\sqrt{-2}, \sqrt{-3}), F=\mathbb{Q}$. Here $p=3$ is an unramified supercuspidal prime of level zero with order $o(\theta(s))=8$, so $\theta(s)+\theta(s)^{3} \neq 0$. We choose $p^{\prime}=19$ and checked that $a_{19}=-4$. Clearly $v_{3}\left(a_{19}^{2} \epsilon^{-1}(19)\right)=0$, so $X_{3}$ is unramified. Note that 3 divides $\operatorname{disc}(E \mid F)$; thus the converse of Corollary 6.3 (c) is false.

(3) $f \in S_{2}(405,[0,2])$ is 3-minimal with $E=\mathbb{Q}(\sqrt{-2}, \sqrt{3})$ and $F=\mathbb{Q} \cdot p=3$ is an unramified supercuspidal prime of positive level, with $o(\theta(s)) \mid 4$, so $\theta(s)+\theta(s)^{3}$ may vanish. But since the level $>0$, we can still apply Theorem 6.2 We choose $p^{\prime}=163$ and checked that $a_{p^{\prime}}^{2} \epsilon^{-1}\left(p^{\prime}\right) \equiv 6 \bmod \mathbb{Q}^{*^{2}}$. Hence $X_{3}$ ramifies.

(4) $f \in S_{2}(99,[3,5]), E=\mathbb{Q}(\sqrt{-2}, \sqrt{3}), F=\mathbb{Q}$. $p=3$ is an unramified supercuspidal prime of level zero with $\theta(s)+\theta(s)^{3} \neq 0$. The order of $3 \in(\mathbb{Z} / 11 \mathbb{Z})^{*}$ is 5 ; hence by Corollary 6.3 (a), $X_{3}$ is unramified.

(5) $f \in S_{2}(99,[0,2]),[E: \mathbb{Q}]=8$ and $F=\mathbb{Q}(\sqrt{5}) . p=3$ is an unramified supercuspidal prime of level zero. The order of $\theta(s)$ is 4 , so $\theta(s)+\theta(s)^{3}=0$, and we cannot apply Theorems 6.1 or 6.2 . Note that $v=3$ is the unique prime in $F$ lying above 3 , and $F_{v}=\mathbb{Q}_{3}(\sqrt{5})=\mathbb{Q}_{3^{2}}$. Thus $f_{v}=2$, but still $\left[X_{v}\right] \sim-1$. This proves the necessity of the condition on $\theta(s)$ in Theorem 6.1

(6) $f \in S_{2}(375,[1,25]),[E: \mathbb{Q}]=16$ and $F=\mathbb{Q}(\sqrt{5}) \cdot p=5 \equiv 1 \bmod 4$ is a ramified supercuspidal prime for $f$, and $K=\mathbb{Q}_{5}(\sqrt{5})$. There is a unique prime $v$ in $F$ above 5 , and $\left[F_{v}: \mathbb{Q}_{5}\right]=2$. As $\epsilon_{p}(-1)^{2\left[F_{v}: \mathbb{Q}_{p}\right] /(p-1)}=-1, X_{v}$ is ramified by Corollary 7.3. In fact, $X_{f}$ is ramified at the primes above 5 , 89 .

(7) $f \in S_{5}(27,[9])$ with $E=\mathbb{Q}(\sqrt{-1})$ and $F=\mathbb{Q} . \quad p=3$ is a ramified supercuspidal prime for $f$. For $p=3, p^{\prime \prime}=53$ satisfies $(* *)$, and we checked that $a_{53}^{2}=-2537649=-1593^{2}$. So $(-1)^{k} a_{p^{\prime \prime}}^{2} \epsilon^{-1}\left(p^{\prime \prime}\right)=a_{53}^{2} \equiv-1$ $\bmod N_{K \mid \mathbb{Q}_{p}} K^{*}$. For any ramified quadratic extension $K$ of $\mathbb{Q}_{3},\left(-1, K \mid \mathbb{Q}_{3}\right)$ $=-1$. Hence by Theorem 7.4, $X_{3}$ is ramified. Note that here $3 \nmid \operatorname{disc}(E \mid F)$, and $N^{\prime}=1$, but still $\left[X_{3}\right] \sim-1$. So the analogue of Corollary 6.3 does not hold in this case.

(8) $f \in S_{5}(27,[9])$ with $E=\mathbb{Q}(\sqrt{-6})$, and $F=\mathbb{Q} . \quad p=3$ is a ramified supercuspidal prime and $K=\mathbb{Q}_{3}(\sqrt{-3})$. We choose $p^{\prime \prime}=53$ as before, and compute $a_{p^{\prime \prime}}^{2}=a_{53}^{2}=-8468064=-6 \cdot 1188^{2}$. Hence $(-1)^{k} a_{p^{\prime \prime}}^{2} \epsilon^{-1}\left(p^{\prime \prime}\right)=$ $a_{p^{\prime \prime}}^{2} \equiv-6 \equiv 3 \bmod N_{K \mid \mathbb{Q}_{3}} K^{*}$. But $\left(3, K \mid \mathbb{Q}_{3}\right)=1$, so $X_{3}$ is unramified by Theorem 7.4. In fact, $X_{f}$ is ramified only at 2 and $\infty$. 
Errata to BG13

(1) Page 517, line 8: The condition " $N_{p} \geq 2>C_{p}$ " should be replaced by " $N_{p} \geq 2, N_{p}>C_{p}$ ".

(2) Page 522 , line 4: In the definition of $m_{v}^{\dagger}$, “[ $\left.F_{v}: \mathbb{Q}_{p^{\dagger}}\right]$ " should be replaced by " $\left[F_{v}: \mathbb{Q}_{p}\right]$ ".

(3) Page 523, line 1: The inequality " $\leq$ " should be replaced by " $<$ ".

(4) Page 539, Prop. 33: One should add the following condition in the hypotheses: "If $K / \mathbb{Q}_{p}$ is unramified, then assume $\chi\left(g_{s}\right)+\chi\left(g_{s}\right)^{p} \neq 0$, where $g_{s} \in G_{K}$ corresponds to a primitive $\left(p^{2}-1\right)$-th root of unity $s \in K^{*}$." Without this assumption, the usual argument referred to on line 16 of page 540 does not work.

\section{REFERENCES}

[AL78] A. O. L. Atkin and Wen Ch'ing Winnie Li, Twists of newforms and pseudo-eigenvalues of $W$-operators, Invent. Math. 48 (1978), no. 3, 221-243, DOI 10.1007/BF01390245. MR508986 (80a:10040)

[BG03] A. F. Brown and E. P. Ghate, Endomorphism algebras of motives attached to elliptic modular forms (English, with English and French summaries), Ann. Inst. Fourier (Grenoble) 53 (2003), no. 6, 1615-1676. MR2038777 (2004m:11089)

[BG13] Debargha Banerjee and Eknath Ghate, Adjoint lifts and modular endomorphism algebras, Israel J. Math. 195 (2013), no. 2, 507-543, DOI 10.1007/s11856-012-0108-y. MR.3096563

[BH06] Colin J. Bushnell and Guy Henniart, The local Langlands conjecture for GL(2), Grundlehren der Mathematischen Wissenschaften [Fundamental Principles of Mathematical Sciences], vol. 335, Springer-Verlag, Berlin, 2006. MR2234120 (2007m:22013)

[CF10] J.W.S. Cassels and A. Fröhlich, Algebraic Number Theory, London Mathematical Society, 2nd edition, 2010.

[GGQ05] Eknath Ghate, Enrique González-Jiménez, and Jordi Quer, On the Brauer class of modular endomorphism algebras, Int. Math. Res. Not. 12 (2005), 701-723, DOI 10.1155/IMRN.2005.701. MR2146605 (2006b:11058)

[LW12] David Loeffler and Jared Weinstein, On the computation of local components of a newform, Math. Comp. 81 (2012), no. 278, 1179-1200, DOI 10.1090/S0025-5718-201102530-5. MR2869056 (2012k:11064)

[Qu98] Jordi Quer, La classe de Brauer de l'algèbre d'endomorphismes d'une variété abélienne modulaire (French, with English and French summaries), C. R. Acad. Sci. Paris Sér. I Math. 327 (1998), no. 3, 227-230, DOI 10.1016/S0764-4442(98)80137-7. MR.1650241 (99j:14045)

[Qu05] J. Quer, Tables of modular endomorphism algebras $X_{f}=\operatorname{End}\left(M_{f}\right)$ of motives $M_{f}$ attached to non-CM newforms, 255 pages, 2005.

[Ri80] Kenneth A. Ribet, Twists of modular forms and endomorphisms of abelian varieties, Math. Ann. 253 (1980), no. 1, 43-62, DOI 10.1007/BF01457819. MR/594532(82e:10043)

[Ri81] K. A. Ribet, Endomorphism algebras of abelian varieties attached to newforms of weight 2, Seminar on Number Theory, Paris 1979-80, Progr. Math., vol. 12, Birkhäuser, Boston, Mass., 1981, pp. 263-276. MR633903 (82m:10044)

[Ri04] Kenneth A. Ribet, Abelian varieties over $\mathbf{Q}$ and modular forms, Modular curves and abelian varieties, Progr. Math., vol. 224, Birkhäuser, Basel, 2004, pp. 241-261, DOI 10.1007/978-3-0348-7919-4_15. MR2058653 (2005k:11120)

Tata Institute of Fundamental Research, Homi Bhabha road, Mumbai 400005, India E-mail address: shalini@math.tifr.res.in

Tata Institute of Fundamental Research, Homi Bhabha road, Mumbai 400005, India

E-mail address: eghate@math.tifr.res.in 